\title{
Density representation of Long's equation
}

\author{
M. Humi \\ Department of Mathematical Sciences, Worcester Polytechnic Institute, 100 Institute Road, Worcester, MA 01609, USA
}

Received: 8 November 2006 - Revised: 1 February 2007 - Accepted: 24 May 2007 - Published: 11 June 2007

\begin{abstract}
Long's equation describes two dimensional stratified atmospheric flow over terrain. Its solutions using regular first order perturbations and linear approximation were investigated analytically and numerically by many authors. Special attention was paid to the properties of the gravity waves that have been predicted to be generated as a result. In this paper we derive a new representation of this equation in terms of the atmospheric density. This new equation is used then to study the steady state that results from some ideal upstream density profiles and the generation of gravity waves. Furthermore we compare the new formulation with the stream function formulation of Long equation and develop new criteria for the stability of the flow.
\end{abstract}

\section{Introduction}

Long's equation (Long, 1953, 1954, 1955, 1959) models the flow of stratified incompressible fluid in two dimensions over terrain. When the base state of the flow (that is the unperturbed flow field far upstream) is without shear the numerical solutions (in the form of steady lee waves) of this equation in various settings and approximations were studied by many authors (Drazin, 1961; Drazin and Moore, 1967; Durran, 1992; Lily and Klemp, 1979; Peltier and Clark, 1983; Smith, 1980, 1989; Yih, 1967; Davis, 1999). The most common approximation in these studies was to set Brunt-Väisälä frequency to a constant or a step function over the computational domain. Moreover the values of the parameters $\beta$ and $\mu$ which appear in this equation were set to zero. In this (singular) limit the nonlinear terms and one of the leading second order derivatives in the equation drop out and the equation reduces to that of a linear harmonic oscillator over two dimensional domain. Careful studies (Lily and Klemp, 1979)

Correspondence to: $\mathrm{M}$. Humi

(mhumi@wpi.edu) showed that these approximations set strong limitations on the validity of the derived solutions (Peltier and Clark, 1983).

Long's equation also provides the theoretical framework for the analysis of experimental data (Shutts et al., 1988; Jumper et al., 2004) under the assumption of shearless base flow. (An assumption which, in general, is not supported by the data). An extensive list of references appears in Baines (1995), Nappo (2002), Yih (1980).

An analytic approach to the study of the solutions of this nonlinear equation and its bifurcations was initiated recently by the current author (Humi, 2004a, 2006). We showed that for a base flow without shear and under rather mild restrictions the nonlinear terms in the equation can be simplified. We also identified the "slow variable" that controls the nonlinear oscillations in this equation. Using phase averaging approximation we derived for self similar solutions of this equation a formula for the attenuation of the stream function perturbation with height. This result is generically related to the presence of the nonlinear terms in Long's equation. The impact that shear has on the generation and amplitude of gravity waves was investigated by us in Humi (2006).

The "Achilles heel" of Long's equation is related to the fact that the value of the Brunt-Väisälä frequency is usually set apriori to a "reasonable constant" which is based on the density profile of the flow in the far upstream. Furthermore there is no feedback to its value (or variation in its value) from the resulting flow field over the computational domain.

In some applications (e.g. determination of the refraction structure parameter, $C_{n}^{2}$; Humi, 2004b) or questions about airplane safety, the atmospheric density distribution is at least as important as the flow field. Furthermore density is one of the primitive variables for model atmospheres and its profile can be used to determine temperature profiles assuming perfect gas law. It follows then that a reformulation of Long's equation in terms of the atmospheric density can offer, in some instances, conceptual and practical advantages over the same formulation in terms of the stream function or

Published by Copernicus GmbH on behalf of the European Geosciences Union and the American Geophysical Union. 
"streamline displacements". This is in spite of the fact that from a mathematical point of view the two formulations are in principle equivalent. Thus from a practical point of view each formulation may turn out to be more useful than the other under some circumstances.

With this motivation it is our objective in this paper to derive a representation of Long's equation in terms of the variable atmospheric density. This representation will require an apriori idealized modeling of the flow field. Thus this new representation of Long's equation stands for the "polar opposite" to the one that was derived originally by Long (1953). We shall use this representation to derive a new stability criteria for the flow. We demonstrate also that it yields new insights about the excitation of gravity waves over topography. To this end we study some idealized density and velocity profiles in the far upstream limit and investigate whether these profiles support the generation of steady state (density) gravity waves over topography.

This density representation provides also an efficient algorithm to simulate flows with specified upstream density and velocity profiles.

The plan of the paper is as follows: in Sect. 2 we present a short review of the usual derivation of Long's equation and some aspects of its solutions. In Sect. 3 we derive the (general) density representation of this equation and specialize its form to some idealized density profiles in the far upstream. In Sect. 4 we consider the choice of the parameter function that appears in the density representation and the related question of gravity wave generation. Section 5 compares the new formulation with the classical stream function formulation of Long's equation. We derive also some (approximate) analytic solutions over low topography. Furthermore we derive a stability criteria for the density distribution and apply it to the idealized density profiles that were studied in previous sections. In Sect. 6 we discuss the results of simulations for several model atmospheres and the effect of some parameters that control the topography on the density distribution. We end up in Sect. 7. with a summary and conclusions.

\section{Long's equation - a short overview}

In two dimensions $(x, z)$ the flow of a steady inviscid and incompressible stratified fluid is modeled by the following equations:

$u_{x}+w_{z}=0$

$u \rho_{x}+w \rho_{z}=0$

$\rho\left(u u_{x}+w u_{z}\right)=-p_{x}$

$\rho\left(u w_{x}+w w_{z}\right)=-p_{z}-\rho g$ where subscripts indicate differentiation with respect to the indicated variable, $\mathbf{u}=(u, w)$ is the fluid velocity, $\rho$ is its density $p$ is the pressure and $g$ is the acceleration of gravity.

We can non-dimensionalize these equations by introducing

$\bar{x}=\frac{x}{L}, \quad \bar{z}=\frac{N_{0}}{U_{0}} z, \quad \bar{u}=\frac{u}{U_{0}}, \quad \bar{w}=\frac{L N_{0}}{U_{0}^{2}} w$

$\bar{\rho}=\frac{\rho}{\bar{\rho}_{0}}, \quad \bar{p}=\frac{N_{0}}{g U_{0} \rho_{0}} p$

where $L$ represents a characteristic length, and $U_{0}, \overline{\rho_{0}}$ represent respectively the free stream velocity and averaged base density (i.e. here $\bar{\rho}_{0}$ is a constant). $N_{0}^{2}$ is an averaged value of the Brunt-Väisälä frequency

$N^{2}=-\frac{g}{\rho_{0}} \frac{d \rho_{0}}{d z}$.

In these new variables Eqs. (1-4) take the following form (for brevity we drop the bars)

$u_{x}+w_{z}=0$

$u \rho_{x}+w \rho_{z}=0$

$\beta \rho\left(u u_{x}+w u_{z}\right)=-p_{x}$

$\beta \rho\left(u w_{x}+w w_{z}\right)=-\mu^{-2}\left(p_{z}+\rho\right)$

where

$\beta=\frac{N_{0} U_{0}}{g}$

$\mu=\frac{U_{0}}{N_{0} L}$.

$\beta$ is the Boussinesq parameter (Davis, 1999) which controls stratification effects (assuming $U_{0} \neq 0$ ) and $\mu$ is the long wave parameter which controls dispersive effects (or the deviation from the hydrostatic approximation). In the limit $\mu=0$ the hydrostatic approximation is fully satisfied (Smith, 1980, 1989).

In view of Eq. (7) we can introduce a stream function $\psi$ so that

$u=\psi_{z}, w=-\psi_{x}$.

Using this stream function we can rewrite Eq. (8) as

$J\{\rho, \psi\}=0$ 
where for any two (smooth) functions $f, g$

$J\{f, g\}=\frac{\partial f}{\partial x} \frac{\partial g}{\partial z}-\frac{\partial f}{\partial z} \frac{\partial g}{\partial x}$

Equation (14) implies that the functions $\rho, \psi$ are dependent on each other and we can express each of them in terms of the other. Thus we can write $\psi$ as $\psi(\rho)$ or $\rho$ as $\rho(\psi)$.

Using $\psi$ the momentum equations (9), (10) become

$\beta \rho\left(\psi_{z} \psi_{z x}-\psi_{x} \psi_{z z}\right)=-p_{x}$

$\beta \rho\left(-\psi_{z} \psi_{x x}+\psi_{x} \psi_{x z}\right)=-\mu^{-2}\left(p_{z}+\rho\right)$

For brevity we eliminate $\mu$ from these equations by the transformation $\bar{x}=\mu x$. This leaves Eq. (16) unchanged and in Eq. (17) $\mu$ cancels out. (In the following we drop the bars on the variable $x$ ).

To eliminate $p$ from Eqs. (16), (17) (after the transformation mentioned above) we differentiate Eqs. (16) and (17) with respect to $z, x$, respectively and subtract. This leads to

$$
\begin{aligned}
\rho_{z}\left(\psi_{z} \psi_{z x}-\psi_{x} \psi_{z z}\right) & +\rho\left(\psi_{z} \psi_{z x}-\psi_{x} \psi_{z z}\right)_{z} \\
& -\rho_{x}\left(-\psi_{z} \psi_{x x}+\psi_{x} \psi_{x z}\right) \\
& -\rho\left(-\psi_{z} \psi_{x x}+\psi_{x} \psi_{x z}\right)_{x} \\
& =-\frac{1}{\beta} J\{z, \rho\}
\end{aligned}
$$

Using Eq. (14) we can rewrite this equation (after some algebra) as

$\rho J\left\{\nabla^{2} \psi, \psi\right\}+J\left\{\frac{1}{2}\left(\psi_{x}^{2}+\psi_{z}^{2}\right), \rho\right\}=-\frac{1}{\beta} J\{z, \rho\}$

However in view of Eqs. (8), $\rho=\rho(\psi)$ and this fact can be used to eliminate $\rho$ from Eq. (19). To this end we observe that

$\rho_{x}=\rho_{\psi} \psi_{x}, \quad \rho_{z}=\rho_{\psi} \psi_{z}$,

This leads then to the following equation for $\psi$ (Davis, 1999)

$\nabla^{2} \psi-N^{2}(\psi)\left[z+\frac{\beta}{2}\left(\psi_{z}^{2}+\psi_{x}^{2}\right)\right]=G(\psi)$

where

$N^{2}(\psi)=-\frac{\rho_{\psi}}{\beta \rho}$

is the nondimensional Brunt-Väisälä frequency. We observe that in this definition $N^{2}$ is a function of $\psi$. (As a result it can be an additional source of nonlinearity in Eq. 21) This is in contrast to the previous definition of this quantity in Eq. (6) which depends only on the base state.
$G(\psi)$ is some unknown function which is determined from the base flow. Henceforth we assume it to be a function of $z$ only. To carry out this determination of $G$ we consider Eq. (21) at $x=-\infty$ and express the left hand side of this equation in terms of $\psi$ only. (Assuming that disturbances do not propagate far upstream (Baines, 1995; Yih, 1980). Equation (21) is referred to as Long's equation.

For example if we let

$\psi(-\infty, z)=z$

i.e. consider a shearless base flow with $u(-\infty, z)=1$ then

$G(\psi)=-N^{2}(\psi)\left(\psi+\frac{\beta}{2}\right)$

and Eq. (21) becomes:

$\nabla^{2} \psi-N^{2}(\psi)\left[z-\psi+\frac{\beta}{2}\left(\psi_{z}^{2}+\psi_{x}^{2}-1\right)\right]=0$.

It is evident from this derivation that different profiles for the base flow at $x=-\infty$ will lead to different forms of $G(\psi)$.

For a general base flow in an unbounded domain over topography with shape $f(x)$ and maximum height $H$ the following boundary conditions are imposed on $\psi$

$\psi(-\infty, z)=\psi_{0}(z)$

$\psi(x, \tau f(x))=$ constant,$\quad \tau=\frac{H N_{0}}{U_{0}}$

where the constant in Eq. (27) is (usually) set to zero. As to the boundary condition on $\psi(\infty, z)$ we observe that Long's equation contains no dissipation terms and therefore only radiation boundary conditions can be imposed in this limit. Similarly at $z=\infty$ it is customary to impose (following Durran, 1992) radiation boundary conditions.

For the perturbation from the shearless base flow

$\eta=\psi-z$

Eq. (25) becomes

$\nabla^{2} \eta-\gamma^{2}\left(\eta_{z}^{2}+\eta_{x}^{2}\right)-N^{2}(z+\eta)\left(\beta \eta_{z}-\eta\right)=0$

where

$\gamma^{2}=\frac{N^{2}(\psi) \beta}{2}$

We observe that when $|\tau| \ll 1$ the boundary condition (27) can be approximated by

$\eta(x, 0)=-\tau f(x)$.

When $N$ is constant Eq. (29) is invariant with respect to translations in $x, z$ and hence admits self-similar solutions of the 
form $\eta=f(k x+m z)$ (Humi, 2004a). These solutions are interpreted as gravity waves that are generated by the flow over the topography.

From a numerical point of view it is a common practice (Durran, 1992; Lily and Klemp, 1979; Davis, 1999) to solve Eq. (29) in the limit $\beta=0$ and $\mu=0$ with constant (or step function) $N$ over the domain (and this value of $N$ remain unchanged even though $\psi$ is variable over the domain. (One can restore $\mu$ to Eq. (29) by the the transformation $\bar{x}=\frac{x}{\mu}$.)

Equation (29) reduces then to a linear equation

$\eta_{z z}+N^{2} \eta=0$.

We observe that the limit $\beta=0$ can be obtained either by letting $U_{0} \rightarrow 0$ or $N_{0} \rightarrow 0$. In the following we assume that this limit is obtained as $U_{0} \rightarrow 0$ (so that stratification persists in this limit).

Equation (32) is a singular limit of Long's equation as one of the leading second order derivatives drops when $\mu=0$ and the nonlinear terms drops out when $\beta=0$. This approximation and its limitations were considered numerically and analytically (Drazin and Moore, 1967; Durran, 1992; Humi, 2004a, 2006) and was found to be justified only under strong restrictions even under the assumption that the base flow is shearless. Nevertheless it is used routinely in the actual analysis of atmospheric data (Shutts et al., 1988; Jumper et al., 2004; Baines, 1995).

\section{Long's equation - density representation}

In view of Eq. (14) we can express $\rho$ in terms of $\psi$ or $\psi$ in terms of $\rho$. Using the first possibility $\rho=\rho(\psi)$ leads to Eq. (21). However if we let $\psi=\psi(\rho)$ and use the following identities

$\psi_{x}=\psi_{\rho} \rho_{x}, \quad \psi_{z}=\psi_{\rho} \rho_{z}, \quad \nabla^{2} \psi=\psi_{\rho \rho}\left[\rho_{x}^{2}+\rho_{z}^{2}\right]+\psi_{\rho} \nabla^{2} \rho$.

then after some algebra we obtain from Eq. (18) the following

$J\left\{\left(\beta \rho \psi_{\rho}^{2}\right) \nabla^{2} \rho+\frac{1}{2}\left(\beta \rho \psi_{\rho}^{2}\right)_{\rho}\left[\rho_{x}^{2}+\rho_{z}^{2}\right]+z, \rho\right\}=0$.

Since for any function of $F(\rho)$ we have $J\{F(\rho), \rho\}=0$. we infer that

$h(\rho) \nabla^{2} \rho+\frac{1}{2} h^{\prime}(\rho)\left[\rho_{x}^{2}+\rho_{z}^{2}\right]+z=S(\rho), \quad h^{\prime}=\frac{d h(\rho)}{d \rho}$.

where

$h(\rho)=\beta \rho \psi_{\rho}^{2}$

and $S(\rho)$ is some function of $\rho$ which is determined by the functional form of $\rho$ in the far upstream boundary. This function can be considered as an integration "constant" or gauge.
Physically it reflects the relationship between $\rho$ and $\psi$ in the far upstream boundary. Equation (35) can be considered to be the "density representation of Long's equation".

We also note that Eq. (35) can be written in the form

$h(\rho)^{1 / 2} \nabla \cdot\left(h(\rho)^{1 / 2} \nabla \rho\right)+z=S(\rho)$.

In Eq. (35) $h(\rho)$ can be considered as a parameter function which is determined by the momentum distribution in the far upstream base flow. This is similar to the definition of the Brunt-Väisälä frequency in Eq. 6).

We now give several examples for the determination of the function $S(\rho)$ and the resulting forms of Eq. (35).

\subsection{Case 1: $\lim _{x \rightarrow-\infty} \rho(x, z)=1-\alpha z$}

(Here and in the following we normalize $\rho(-\infty, 0)$ to 1 and $\alpha$ is a constant.)

Substituting this value of $\rho$ in Eq. (35) and expressing $z$ as $z=\frac{1-\rho}{\alpha}$ we obtain

$S(\rho)=\frac{\alpha^{2}}{2} h^{\prime}(\rho)+\frac{1-\rho}{\alpha}$.

Equation (35) becomes then

$h(\rho) \nabla^{2} \rho+\frac{1}{2} h^{\prime}(\rho)\left[\rho_{x}^{2}+\rho_{z}^{2}-\alpha^{2}\right]-\frac{1-\rho}{\alpha}+z=0$

3.2 Case 2: $\lim _{x \rightarrow-\infty} \rho(x, z)=e^{-\alpha z}$

This is the case of an isothermal atmosphere with $\alpha=\frac{g}{R T_{0}}$ (Dutton, 1986, p. 69).

For this base state we have

$S(\rho)=\alpha^{2}\left[h(\rho) \rho+\frac{1}{2} h^{\prime}(\rho) \rho^{2}\right]-\frac{\ln \rho}{\alpha}$

and the density representation of Long's equation becomes

$h(\rho)\left[\nabla^{2} \rho-\alpha^{2} \rho\right]+\frac{1}{2} h^{\prime}(\rho)\left[\rho_{x}^{2}+\rho_{z}^{2}-\alpha^{2} \rho^{2}\right]+\frac{\ln \rho}{\alpha}+z=0$

3.3 Case 3: $\lim _{x \rightarrow-\infty} \rho(x, z)=(1-\alpha z)^{r}, \quad r \neq 1$

For proper values of $\alpha$ and $r$ this relationship has been used for convective atmospheric studies (Dutton, 1986, p. 67-69).

Following the same procedure outlined above we have

$S(\rho)=\alpha^{2} r(r-1) h(\rho) \rho^{1-2 / r}+\frac{1}{2} \alpha^{2} r^{2} h^{\prime}(\rho) \rho^{2-2 / r}+\frac{1-\rho^{1 / r}}{\alpha}$

and Eq. (35) becomes

$$
\begin{aligned}
h(\rho) & {\left[\nabla^{2} \rho-\alpha^{2} r(r-1) \rho^{1-2 / r}\right] } \\
+ & \frac{1}{2} h^{\prime}(\rho)\left[\rho_{x}^{2}+\rho_{z}^{2}-\alpha^{2} r^{2} \rho^{2-2 / r}\right] \\
& -\frac{1-\rho^{1 / r}}{\alpha}+z=0
\end{aligned}
$$




\section{Equations for perturbations from the base state}

In this section we derive the equations for the perturbation $\eta=\rho-\rho_{0}$ (where $\rho_{0}=\lim _{x \rightarrow-\infty} \rho(x, z)$ ) from different profiles of the base density. We would like to emphasize however that although we refer to $\eta$ as a perturbation it is more appropriate to refer to it as "deviation from base state" as we place no restriction on the size of $\eta$. Furthermore the equations we derive for $\eta$ are exact (and nonlinear) in general. These equations are useful for the numerical simulation of the density over topography and for the analytical study of gravity wave generation from different base state.

We consider again the three cases that were discussed in the previous section.

Case 1:

Substituting for $\rho$ in Eq. (39) yields

$h(\rho) \nabla^{2} \eta+\frac{1}{2} h^{\prime}(\rho)\left[\eta_{x}^{2}+\eta_{z}^{2}-2 \alpha \eta_{z}\right]+\frac{1}{\alpha} \eta=0$.

Here (and in the following) $h$ and its derivative have to be expressed in terms of $\eta$ i.e. $h=h\left(\rho_{0}+\eta\right)$ however we shall leave the notation unchanged for brevity. Furthermore there is no chance of confusion. We wish to emphasize again that Eq. (44) is an exact equation for $\eta$.

When $\psi_{0}=\lim _{x \rightarrow-\infty} \psi(x, z)=z$ (that is the base flow is shearless) then $\psi_{0}=\frac{1-\rho_{0}}{\alpha}$ and it follows that

$h(\rho)=\frac{\beta \rho}{\alpha^{2}}$

Similarly if $\psi_{0}=z^{2}$ (Shear flow with $u=2 z$ ) then

$h(\rho)=\frac{4 \beta \rho(1-\rho)^{2}}{\alpha^{4}}$

Here we assume that the functional form of $h$ remains unchanged throughout the region under consideration.

From this discussion we infer that Eq. (44) will remain invariant under translations in $x, z$ and therefore admits selfsimilar solutions of the form of $\eta=f(k x+m z)$ only if we can approximate $h$ and $h^{\prime}$ by constants. (That is we first evaluate $h^{\prime}$ using the exact expression for $h(\rho)$ and then approximate it by a constant). One possible strategy to accomplish this will be to approximate these functions by $h\left(\rho_{0}\right)$ and $h^{\prime}\left(\rho_{0}\right)$ and then replace these functions by a "proper" constant.

For the case given by Eq. (45) such an approximation is possible if $|\alpha z| \ll 1$ in the domain under consideration. We then have $h \approx \frac{\beta}{\alpha^{2}}$ and $h^{\prime}=-\frac{\beta}{\alpha}$. With this additional approximation the resulting equation for $f$ is

$h\left(k^{2}+m^{2}\right) f^{\prime \prime}+\frac{1}{2} h^{\prime}\left[\left(k^{2}+m^{2}\right)\left(f^{\prime}\right)^{2}-2 \alpha m f^{\prime}\right]+\frac{1}{\alpha} f=0$

(where we removed the $\rho$ dependence on $h, h^{\prime}$ to emphasize the fact that these should be considered as constants)

In view of the need for this second approximation it is interesting to determine when $h$ is actually constant. From the definition of $h$ it is easy to see that this will happen when $\psi \sim \rho^{1 / 2}$. For the present base density profile we then have $\psi_{0} \sim(1-\alpha z)^{1 / 2}$ which in turn implies that $u_{0} \sim(1-\alpha z)^{-1 / 2}$ i.e. $u_{0}$ is increasing (slowly) with height. For this special case $h^{\prime}=0$ and the equation for $\eta$ reduces to a linear equation.

$h \nabla^{2} \eta+\frac{1}{\alpha} \eta=0$.

(which can be solved by standard methods). We stress that this an exact result and no approximations (or linearizations) were made in the derivation of this equation.

The same result can be obtained using the formulation of Long's equation in terms of streamline displacements $\delta=z-z_{0}$ (Eq. 5.3.7 in Baines, 1995),

$\nabla^{2} \delta+\frac{1}{q} \frac{d q}{d z}\left(\frac{\partial \delta}{\partial z}-\frac{1}{2}|\nabla \delta|^{2}\right)-\frac{g}{2 q} \frac{\partial \rho_{0}}{\partial z_{0}} \delta=0$.

where $q=\rho_{0} U_{0}^{2} / 2$. In this case a short calculation shows that $q=\alpha^{2} / 8$ i.e. $q$ is a constant and Eq. (49) reduces to a linear equation. This demonstrates again that the traditional and the density formulation Long's equations are equivalent from a mathematical point of view.

\section{Case 2:}

The exact equation for the perturbation $\eta$ in this case is

$$
\begin{gathered}
h(\rho)\left[\nabla^{2} \eta-\alpha^{2} \eta\right]+\frac{1}{2} h^{\prime}(\rho)\left[\eta_{x}^{2}+\eta_{z}^{2}-\alpha^{2} \eta-2 \alpha e^{-\alpha z}\left(\alpha \eta+\eta_{z}\right)\right] \\
+\frac{\ln \left(1+e^{\alpha z} \eta\right)}{\alpha}=0
\end{gathered}
$$

For shearless base flow with $\psi_{0}=z$ we have

$h(\rho)=\frac{\beta}{\alpha^{2} \rho}$.

Similarly for $\psi_{0}=z^{2}$ we obtain

$h(\rho)=\frac{4 \beta(\ln \rho)^{2}}{\alpha^{4} \rho}$.

Equation (50) is not invariant with respect to translations and hence in general will not admit self-similar solutions of the form $\eta=f(k x+m z)$. However, for $|\alpha z| \ll 1$ we can use the approximation $e^{\alpha z} \approx 1$ and under this restriction self-similar solutions will be admitted by the resulting (approximate) equation if in addition $h$ and $h^{\prime}$ are approximated by a constant. The derived equation for $f$ under these approximations is

$$
\begin{aligned}
& h\left[\left(k^{2}+m^{2}\right) f^{\prime \prime}-\alpha^{2} f\right]+\frac{1}{2} h^{\prime}\left[\left(k^{2}+m^{2}\right)\left(f^{\prime}\right)^{2}-\alpha^{2} f-2 \alpha\left(\alpha f+m f^{\prime}\right)\right] \\
& \quad+\frac{\ln (1+f)}{\alpha}=0
\end{aligned}
$$

For $h$ to actually be a constant for this base density profile $\psi$ must satisfy $\psi \sim e^{-\frac{\alpha z}{2}}$ which implies that $u=\psi_{z}$ decays exponentially with height. The resulting equation for $\eta$ is

$h\left[\nabla^{2} \eta-\alpha^{2} \eta\right]+\frac{\ln \left(1+e^{\alpha z} \eta\right)}{\alpha}=0$ 
which is still nonlinear. However if we assume that $\left|e^{\alpha z} \eta\right|$ $\ll 1$ we can linearize this equation to obtain

$h\left[\nabla^{2} \eta-\alpha^{2} \eta\right]+\frac{e^{\alpha z} \eta}{\alpha}=0$

Case 3:

The exact equation for $\eta$ in this case is

$$
\begin{aligned}
& h(\rho)\left\{\nabla^{2} \eta+\alpha^{2} r(r-1)(1-\alpha z)^{r-2}\left[1-B^{1-2 / r}\right]\right\}+ \\
& \frac{1}{2} h^{\prime}(\rho)\left\{\left[\eta_{x}^{2}+\left[\eta_{z}-\alpha r(1-\alpha z)^{r-1}\right]^{2}\right.\right. \\
& \left.-\alpha^{2} r^{2}(1-\alpha z)^{2 r-2} B^{2-2 / r}\right\}+\frac{1-\alpha z}{\alpha}\left[B^{1 / r}-1\right]=0
\end{aligned}
$$

where

$$
B=1+\frac{\eta}{(1-\alpha z)^{r}}
$$

When $\psi_{0}=z$ we have $\psi_{0}=\frac{1-\rho_{0}^{1 / r}}{\alpha}$ which yields

$h(\rho)=\frac{\beta \rho^{(2-r) / r}}{\alpha^{2} r^{2}}$

We see that when $r=2, h$ is a constant.

We can simplify Eq. (56) if $\left|\frac{\eta}{(1-\alpha)^{r}}\right| \ll 1$ using the approximation $(1+s)^{m} \approx 1+m s$ to obtain

$$
\begin{aligned}
& h(\rho)\left\{\nabla^{2} \eta-\alpha^{2}(r-1)(r-2)(1-\alpha z)^{-2} \eta\right\}+ \\
& \frac{1}{2} h^{\prime}(\rho)\left\{\left[\eta_{x}^{2}+\eta_{z}^{2}-2 \alpha r(1-\alpha z)^{r-1} \eta_{z}-2 \alpha^{2} r\right.\right. \\
& \left.(r-1)(1-\alpha z)^{r-2} \eta\right\}+\frac{(1-\alpha z)^{1-r} \eta}{r \alpha}=0
\end{aligned}
$$

We see that even under this approximation the equation is not invariant under translations with respect to $z$ and therefore (strictly speaking) does not admit solutions of the form $f(k x+m z)$. However if $|\alpha z| \ll 1$ in the domain under consideration we can make the (additional) approximation $1-\alpha z \approx 1$ and Eq. (58) becomes invariant under translations in $x, z$. Under these approximations a solution of the form $f(k x+m z)$ must satisfy the following equation

$$
\begin{aligned}
& h(\rho)\left[\left(k^{2}+m^{2}\right) f^{\prime \prime}-\alpha^{2}(r-2)(r-1) f\right]+\frac{h^{\prime}(\rho)}{2} \\
& {\left[\left(k^{2}+m^{2}\right)\left(f^{\prime}\right)^{2}-2 m r \alpha f^{\prime}-2 \alpha^{2} r(r-1) f\right]+\frac{f}{r \alpha}=0}
\end{aligned}
$$

When $h$ is a constant and $r=2$, Eq. (56) becomes

$h \nabla^{2} \eta+\frac{1-\alpha z}{\alpha}\left[\sqrt{1+\frac{\eta}{(1-\alpha z)}}-1\right]=0$.

For $|\eta| \ll 1$ we can linearize this equation to obtain

$h \nabla^{2} \eta+\frac{\eta}{2 \alpha(1-\alpha z)}=0$.

\section{Comparisons, solutions and stability}

It is easy to see that the stream-function formulation of Long's equation remains nonlinear for any (nontrivial) base state profiles of $\psi$ and $\rho$. In fact this equation becomes linear only in the limits $\beta \rightarrow 0$ and $\mu \rightarrow 0$ (and constant $N^{2}$ ). These are singular limits from both physical and mathematical points of view which limit severely the insights that can be derived form the linearized form of this equation. In the density formulation even if one linearizes the equation for the deviation $\eta$ from the base state (i.e. assume $|\eta| \ll 1$ ) there is no need to impose these limits on $\beta$ and $\mu$. Furthermore we demonstrated in the previous section that for each of the three model atmospheres considered there exist base state profiles of the density and stream function under which the density representation of Long's equation becomes either linear or "mildly" nonlinear and hence amenable to analytic treatment.

For case 1 where $\lim _{x \rightarrow-\infty} \rho(x, z)=(1-\alpha z)$ Eq. (48) is exactly linear regardless of the amplitude of $\eta$. Its eigensolutions (subject to obvious physical constraints) are

$\eta_{\omega}=e^{-\omega z}(A(\omega) \cos v x+B(\omega) \sin v x)$

where $v^{2}=\omega^{2}+\frac{1}{h \alpha}$ and $\omega \geq 0$.

The corresponding stream-function Long's equation (Eq. 21) for this problem with $\psi=\rho^{1 / 2}$ is

$\psi \nabla^{2} \psi+\frac{2}{\beta}\left[z+\frac{\beta}{2}\left(\psi_{x}^{2}+\psi_{z}^{2}\right)-\frac{1-\psi^{2}}{\alpha}\right]=0$.

which is highly nonlinear. However there may exist reverse situations where the traditional formulation of Long's equation in terms of the stream function might be simpler to the one in terms of of the density.

For the other atmospheric models that were considered in the previous section Eqs. (55) and (61) are also linear. (These were derived, however, under the constraint of small perturbation). When $\eta$ is not small the corresponding Eqs. (54), (60) are "weakly" nonlinear. (The square root and log functions damp the nonlinear effects).

From another point of view Eq. (32) with $N=$ constant (which has been used routinely in the literature) admits always solutions of the form $f(k x+m z)$. On the other hand the equations derived for $\eta$ in our formulation show that this form of solution exists only under very special circumstances. In fact even the linearized Eqs. (55), (61) do not admit these solutions as they are not invariant under translations in $z$. This demonstrates that there exist many profiles of base density and stream function for which gravity waves solutions of the form $f(k x+m z)$ (for the density) do not exist.

In addition even though Eqs. (55), (61) have non-constant coefficients they admit eigensolutions in terms of well known functions. Using separation of variables we obtain for the 
nonsingular eigensolutions of Eq. (55)

$\eta_{\omega}(x, z)=J_{k}(s)[A \cos \omega x+B \sin \omega x]$

where

$k=\frac{2 \sqrt{\alpha^{2}+\omega^{2}}}{\alpha}, \quad s=\frac{2 e^{\alpha z / 2}}{\sqrt{h \alpha^{3}}}$.

$J_{k}$ is Bessel functions of the first kind and $\omega$ a real nonnegative parameter. From this representation of the eigensolutions we infer that excitations governed by this equation will decay with $z$ (i.e. height) due to the properties of the Bessel functions.

Similarly for the eigensolutions of Eq. (61) we obtain for $\omega \neq 0$

$$
\begin{aligned}
\eta_{\omega}(x, z)= & (1-\alpha z) e^{\omega z} \operatorname{Kummer} M(n, 2, s) \\
& {[A(\omega) \cos \omega x+B(\omega) \sin \omega x] }
\end{aligned}
$$

and

$$
\eta_{0}(x, z)=A(0) \sqrt{1-\alpha z} J_{1}(r)
$$

for $\omega=0$. In these equations

$n=1-\frac{1}{\beta \omega}, \quad s=\frac{2 \omega}{\alpha}(1-\alpha z), \quad r=\sqrt{\frac{8(1-\alpha z)}{\alpha \beta}}$

and Kummer $M$ is the Kummer-M function (Abramowitz and Stegun, 1970, p. 504).

\subsection{Boundary conditions and analytic solutions}

The eigenfunctions for Eqs. (48), (55), (60) that were derived above enable us to write analytic solutions for these equations over low topography.

To derive the appropriate boundary conditions on $\eta$ to solve Eq. (48) in the presence of topography $\tau f(x)$ we let $\rho=\psi^{2}$ (that is we let the constant of proportionality to be 1). Hence

$$
\begin{aligned}
\eta(x, \tau f(x)) & =\rho(x, \tau f(x))-\rho_{0}(x, \tau f(x)) \\
& =\psi^{2}(x, \tau f(x))-\psi_{0}^{2}(x, \tau f(x))
\end{aligned}
$$

However on the ground (we let) $\psi(x, \tau f(x))=1$. Therefore

$$
\eta(x, \tau f(x))=\alpha \tau f(x)
$$

Observe that this is an exact boundary condition. For low lying topography we can approximate this equation by

$\eta(x, 0)=\alpha \tau f(x)$

This is an approximation similar to the one used in Eq. (31). However we emphasize the fact that in our formulation $\beta$ and $\mu$ are not set to zero. Thus this linearization of the problem is not equivalent to the one that led to Eq. (32).
Following the same procedure outlined in Sect. 2 we obtain for the boundary conditions on Eqs. (54) and (55)

$\eta(x, \tau f(x))=1-e^{-\alpha \tau f(x)}$.

Similarly for Eqs. (60) and (61) we have

$\eta(x, \tau f(x))=\alpha \tau f(x)[2-\alpha \tau f(x)]$.

The general solution of Eq. (48) in the upper half plane is

$\eta(x, z))=\int_{0}^{\infty} e^{-\omega z}(A(\omega) \cos \nu x+B(\omega) \sin \nu x) d \omega$.

For low topography the boundary condition (69) yields then

$A(\omega)=\frac{\alpha \tau}{\pi} \int_{-\infty}^{\infty} f(x) \cos v x d x$,
$B(\omega)=\frac{\alpha \tau}{\pi} \int_{-\infty}^{\infty} f(x) \sin v x d x$.

Similar expressions can be derived for the solutions of Eqs. (55), (61). We wish to emphasize that the only approximation made in the derivation of the solution (72) is due to the topography (or more precisely the boundary condition on the topography) where Eq. (68) was approximated by Eq. (69).

\subsection{Stability}

Assuming that

$\lim _{x \rightarrow-\infty} \rho(x, z)=\rho_{0}(z)$

we infer from Eq. (35) that in this limit $S(\rho)$ must satisfy

$\left.S\left(\rho_{0}\right)=h\left(\rho_{0}\right)\left(\rho_{0}\right)_{z z}+\frac{1}{2} h^{\prime}\left(\rho_{0}\right)\left(\rho_{0}\right)_{z}\right)^{2}+z$

where $z$ on the right hand side of this equation has to be reexpressed in terms of $\rho_{0}$.

To examine the (linear) stability of the $\rho(x, z)$ against a perturbation from the base state we write

$\rho(x, z)=\rho_{0}(z)+\eta(x, z)$.

and substitute this expression in Eq. (35). Linearizing the resulting equation (around $\rho_{0}(z)$ ) and using Eq. (74) we obtain

$$
\begin{gathered}
\nabla^{2} \eta+\frac{h^{\prime}\left(\rho_{0}\right)}{h\left(\rho_{0}\right)}\left(\rho_{0}\right)_{z} \eta_{z}+\frac{1}{h\left(\rho_{0}\right)}\left[h^{\prime}\left(\rho_{0}\right)\left(\rho_{0}\right)_{z z}\right. \\
\left.+\frac{h^{\prime \prime}\left(\rho_{0}\right)}{2}\left(\rho_{0}\right)_{z}^{2}-S^{\prime}\left(\rho_{0}\right)\right] \eta=0
\end{gathered}
$$

where primes demote differentiation with respect to $\rho$.

Since $\rho_{0}$ is a function of $z$ only this equation is separable we can deduce the properties of its solutions by applying separation of variables. Introducing

$\eta(x, z)=\chi(x) \phi(z)$ 
we obtain

$\chi(x)_{x x}+\lambda \chi(x)=0$

and

$$
\begin{aligned}
& h\left(\rho_{0}\right) \phi_{z z}+h^{\prime}\left(\rho_{0}\right)\left(\rho_{0}\right)_{z} \phi_{z}-\left[\lambda+S^{\prime}\left(\rho_{0}\right)-h^{\prime}\left(\rho_{0}\right)\left(\rho_{0}\right)_{z z}\right. \\
& \left.-\frac{h^{\prime \prime}\left(\rho_{0}\right)}{2}\left(\rho_{0}\right)_{z}^{2}\right] \phi=0
\end{aligned}
$$

where $\lambda(>0)$ is the separation of variables constant. Equation (79) can be rewritten as

$\left(h\left(\rho_{0}\right) \phi_{z}\right)_{z}-H(z) \phi=0$

where

$H(z)=\lambda+S^{\prime}\left(\rho_{0}\right)-h^{\prime}\left(\rho_{0}\right)\left(\rho_{0}\right)_{z z}-\frac{h^{\prime \prime}\left(\rho_{0}\right)}{2}\left(\rho_{0}\right)_{z}^{2}$

Equations (80-81) demonstrate that the properties of the perturbation as a function of $z$ depend only on $\lambda$ (i.e. the square of the wave number in the $\mathrm{x}$-direction) and the base state of the flow.

Multiplying Eq. (80) by $\phi(z)$ and integrating by parts we obtain

$\left.h\left(\rho_{0}\right) \phi \phi_{z}\right|_{0} ^{z}-\int_{0}^{z} h\left(\rho_{0}\right) \phi_{z}^{2} d z-\int_{0}^{z} H(z) \phi^{2} d z=0$

Assuming that $\phi(0)=0$ we can rewrite this equation as

$\frac{d \phi^{2}}{d z}=\frac{1}{h\left(\rho_{0}\right)}\left[\int_{0}^{z} h\left(\rho_{0}\right) \phi_{z}^{2} d z+\int_{0}^{z} H(z) \phi^{2} d z\right]$

It follows then that a sufficient condition for the amplitude of $\phi$ to increase with height is

$h\left(\rho_{0}\right)>0, H(z)>0$.

In fact from its definition (Eq. 36) it is clear that $h\left(\rho_{0}\right)$ is always positive. Therefore we need to check only the second condition.

To make further progress we consider now each of the three cases which were discussed in Sect. 3 with $\psi_{0}=z$.

\section{Case 1}

In this case $h(\rho)$ and $S(\rho)$ are given by Eqs. (45), (38), respectively, and we obtain that

$H=\lambda-\frac{1}{\alpha}$

Hence the amplitude of the perturbation will increase whenever $\alpha \lambda>1$. Furthermore we can solve Eq. (80) explicitly in this case to obtain

$\phi(z)=C_{1} J_{0}(w)+C_{2} Y_{0}(w)$

where $C_{1}, C_{2}$ are constants and

$w=2 \sqrt{\frac{(1-\lambda \alpha)(1-\alpha z)}{\alpha \beta}}$
Since $\rho_{0}=1-\alpha z>0$ both $J_{0}$ and $Y_{0}$ will remain bounded if $\alpha \lambda<1$. We conclude then that the amplitude of $\phi$ will remain bounded with height if this condition is satisfied.

\section{Case 2}

Using Eqs. (40), (51) we obtain that

$H(z)=\lambda-\frac{1}{\alpha} e^{\alpha z}$

Hence the amplitude of $\phi$ will increase with height if $\lambda \alpha e^{-\alpha z}>1$.

\section{Case 3}

We use Eqs. (42), (57) and consider only the case with $r=2$. This leads to

$H(z)=\lambda-\frac{1}{2 \alpha(1-\alpha z)}$

Hence the amplitude of $\phi$ will increase with height if

$2 \lambda \alpha(1-\alpha z)>1$.

(The explicit solution of Eq. (80) in this case can be expressed in terms of Kummar functions).

\section{Numerical simulations for the perturbation}

In previous sections we discussed from an analytical point of view different aspects of the density representation of Long's equation for different asymptotic profiles of $\rho$ and $\psi_{0}$ and derived differential equations for the perturbation from the base state. In this section we perform numerical simulations of these equations (that is Eqs. 44, 50, 56) over topography to examine the different flow patterns that are predicted by these equations and their relation to gravity waves. In all cases we considered only a shearless base flow i.e. $\psi_{0}=z$. We also let $r=2$ in case 3, i.e. we solve Eq. (60) (which is a special case of Eq. 56).

To solve for the perturbation $\eta$ over a finite two dimensional domain $[a, c] \times[\tau f(x), b]$ we assumed that $\eta=0$ at $x=a$. Radiation boundary conditions were imposed at $x=c$ and $z=b$. These are necessary to avoid reflection of the outgoing wave. To implement these boundary conditions we used "sponge boundaries" at $x=c$ and $z=b$ with exponential damping (as is done in the NCAR/MM5 mesoscale model (Haggenson et al., 1994, and others). In the simulations that we describe below we let (in nondimensional units) $a=-50$, $b=50$ and $c=50$. The sponge layers start at $z=45$ and $x=45$. In addition we let

$\frac{\partial \eta}{\partial x}=0$ at $x=c ; \quad \frac{\partial \eta}{\partial z}=0$ at $z=b$

Furthermore due to the fact that in these simulations some waves appear to propagate upstream we added a sponge layer at $x=a$ to avoid wave reflection at this boundary. This sponge layer extends up to $x=-45$. 


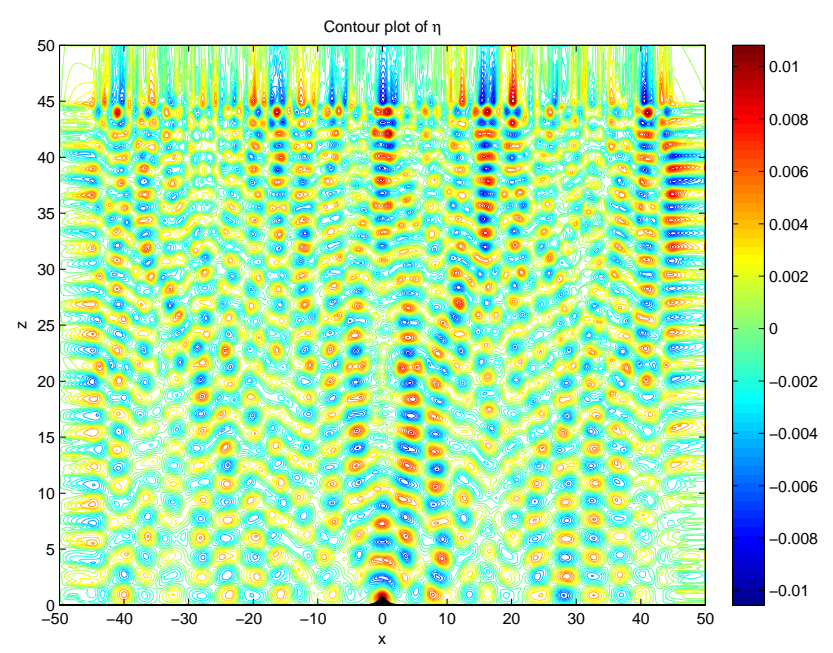

Fig. 1. Contour plot of $\eta$ with $\rho_{0}(x, z)=1-\alpha z, \psi_{0}=z, \tau=0.75$.

To derive the exact boundary condition appropriate for each of the equations mentioned above at the bottom topography we use the same procedure outlined in Sect. 5.1. Letting $\psi(x, \tau f(x))=0$ we obtain the following conditions

Case 1:

$$
\begin{aligned}
\eta(x, \tau f(x)) & \left.=\rho(x, \tau f(x))-\rho_{0}(x, \tau f(x))\right) \\
& =(1-\alpha \psi(x, \tau f(x)))-\left(1-\alpha \psi_{0}(x, \tau f(x))\right. \\
& =\alpha \tau f(x)
\end{aligned}
$$

Case 2:

$\eta(x, \tau f(x))=e^{-\alpha \psi(x, \tau f(x))}-e^{-\alpha \psi_{0}(x, \tau f(x))}=1-e^{-\alpha \tau f(x)}$

Case 3:

$$
\begin{aligned}
\eta(x, \tau f(x)) & =(1-\alpha \psi(x, \tau f(x)))^{2}-\left(1-\alpha \psi_{0}(x, \tau f(x))^{2}\right. \\
& =\alpha \tau f(x)(2-\alpha \tau f(x))
\end{aligned}
$$

To solve Eqs. (44), (50) and (60) we used the software package "FlexPde" $(2006)^{1}$. This is a finite element package (with dynamic local grid refinement) which can simulate a system of (nonlinear) partial differential equations in two dimensions over arbitrary domain. Accordingly, exact boundary conditions were used at the bottom topography. (There was no need to use approximations similar to those in Eq. 69). The convergence criteria for the iterations was that the step error of $\left\|\rho_{m+1}-\rho_{m}\right\|$ was less than $5.10^{-5}$ where $m$ is the iteration number. Due to the fact that dynamic grid refinement was used the total number of (Newton) iterations (approximately 100-125) does not convey the actual computational effort needed to achieve this convergence. However we note that the total number of nodes in the final mesh(es)

\footnotetext{
1 "FlexPde 5" is a trademark of "Pde Solutions Inc.", Antioch,
} CA, 2006.

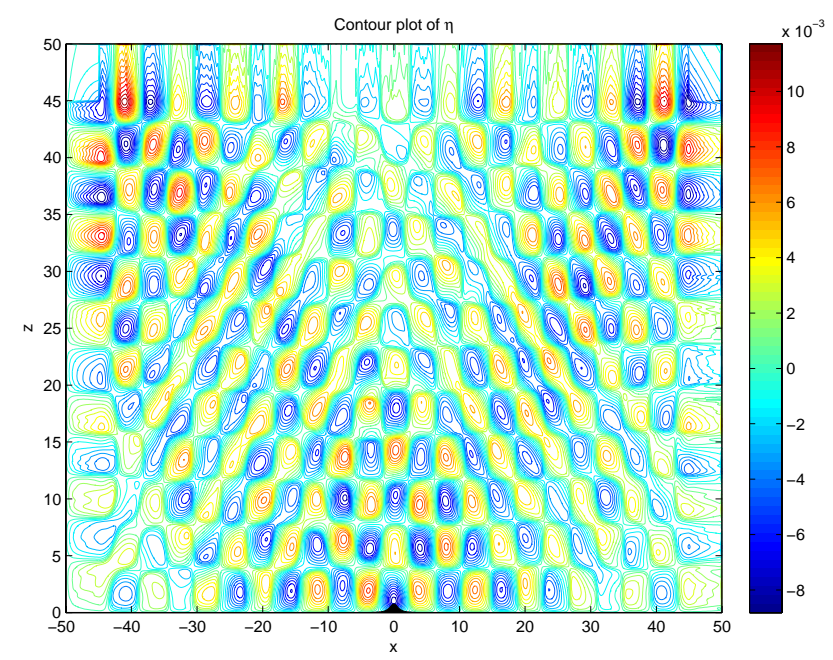

Fig. 2. Contour plot of $\eta$ with $\rho_{0}=e^{-\alpha z}, \psi_{0}=z, \tau=0.75$.

was approximately $5 \times 10^{5}$ and each simulation took about $20-25 \mathrm{~h}$ on a Xeon 3.2 Mhz CPU.

Figures 1, 2, 3 present (respectively) the results of the simulations of the equations mentioned above with these boundary conditions and the following topography shape function

$f(x)=\frac{1}{\left(1+x^{2}\right)^{3 / 2}}$.

The parameters used in these simulations were:

$\tau=0.75, \quad \alpha=0.01, \quad \beta=4.10^{-3}$,

(In case $1, \alpha$ was set to 0.015 to induce stronger stratification).

We see from these figures that in the first two cases there is a clear evidence of gravity waves over the topography. However in the third case there is no such evidence and the picture is completely different. This might explain in part why some current models over predict the production of gravity waves (Eckermann and Preusse, 1999; Dewan et al., 1998). This suppression effect is due to the different base density profile and the nonlinearity of Long's equation. In fact it was demonstrated in Humi (2006) that some shear profiles in the base flow might have the same effect on the generation of gravity waves over topography. Another possible explanation is that the wave spectrum is concentrated at intrinsic frequencies above $N$. The question as to which base profiles have this effect remains an open problem.

We note the difference in the wave pattern in Fig. 2 as compared to Fig. 1 and the difference in their horizontal wave number. We observe also that as expected (due to energy conservation) the amplitude of the waves increases with height due to stratification.

These figures demonstrate clearly the role of the base state and stratification on the generation of gravity waves and their 


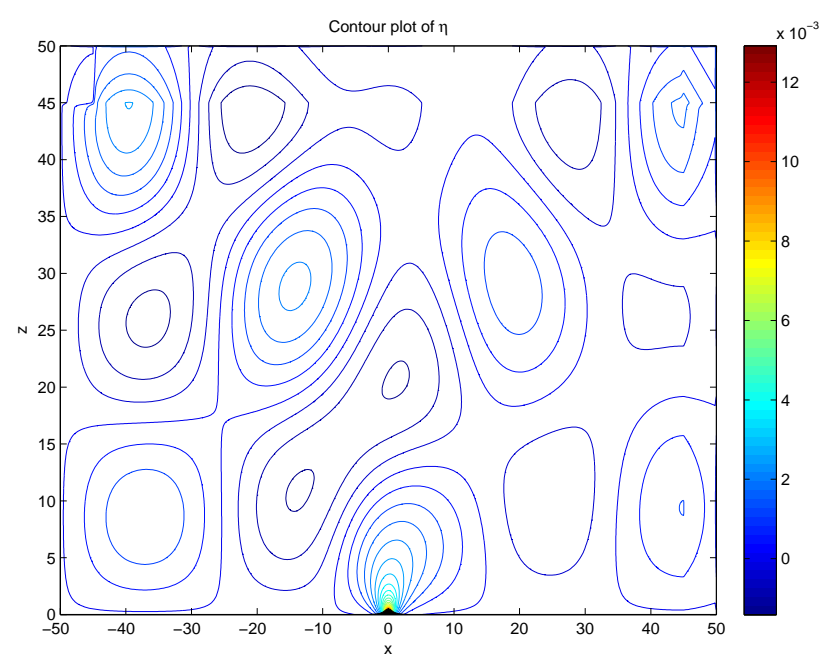

Fig. 3. Contour plot of $\eta$ with $\rho_{0}(x, z)=(1-\alpha z)^{2}, \psi_{0}=z, \tau=0.75$.

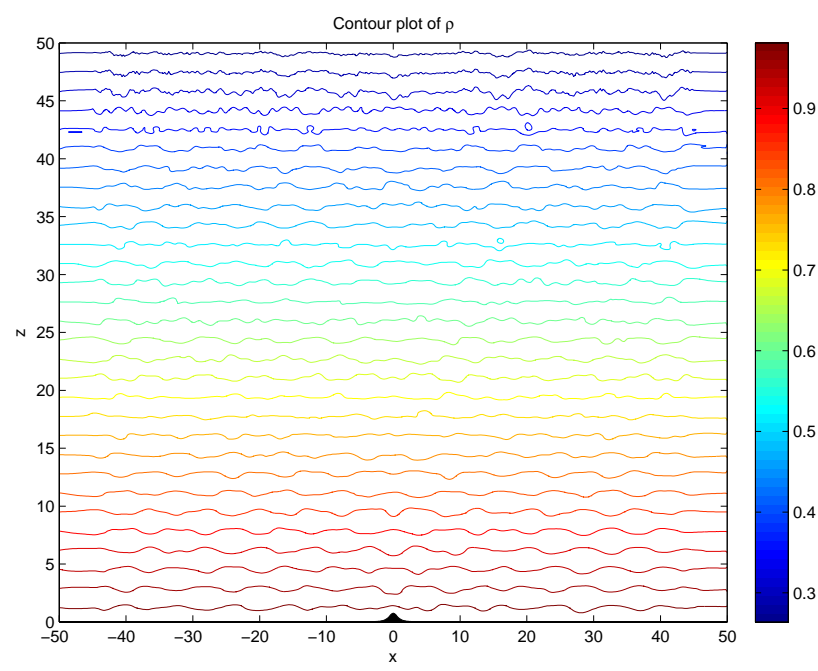

Fig. 4. Contour plot of $\rho$ with $\rho_{0}=1-\alpha z, \psi_{0}=z, \tau=0.75$.

energy. We wish to note the fact that these simulations were carried out with nonzero values of $\beta$ and $\mu$ while most of the simulations carried out in the literature using the streamfunction formulation of Long's equation set these parameters to zero.

We would like to emphasize that in Figs. 1-3 we plotted the perturbation from the base state. In many plots that appear in the literature only the (total) stream function is plotted. To show that the results of our simulations actually have the same general features as those that appeared in the literature we plotted $\rho$ (that is the total density) in Figs. 4, 5. These plots correspond to the perturbations shown in Figs. 1, 2.

Note that in Fig. 2 and to some extent also in Fig. 1 the perturbation propagates in the upstream direction. We attribute

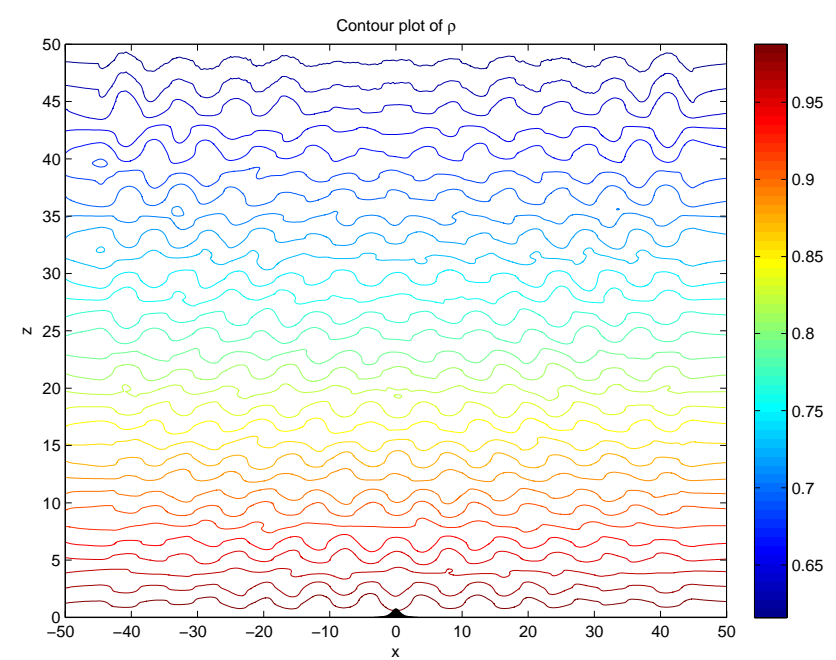

Fig. 5. Contour plot of $\eta$ with $\rho_{0}=e^{-\alpha z}, \psi_{0}=z, \tau=0.75$.

this phenomena (which is absent in some plots that appeared in the literature) to the fact that in our simulations we used nonzero values for $\beta$ and $\mu$ while the figures that appear in the literature usually set these parameters to zero. Furthermore we observe that Long's equation contains no viscosity terms. Hence any decay in the amplitude of the waves is due solely to nonlinear effects. It can be expected therefore that under these settings a perturbation will decay only slowly both downstream and upstream.

\section{$7 \quad$ Summary and conclusions}

We derived in this paper the density analog representation of Long's equation and examined its application to several idealized base density profiles. In this representation the conditions on the base density and flow profiles which support the generation of gravity waves become more transparent. Thus the stream function Long's equation predicts that gravity waves will be generated whenever Brunt-Väisälä frequency can be set to a constant and the base flow is shearless. Due to its complexity no other choices of these parameters was considered in the literature. In the new representation a richer structure emerges which involve both the base density and flow. As a result the properties of different atmospheric structures can be investigated in depth. Using this formalism we derived a new stability criteria for the flow and applied it to three different idealized cases. We also showed that in some instances the new representation of Long's equation lead to analytic solutions for the density distribution.

Edited by: R. Grimshaw

Reviewed by: three referees 


\section{References}

Abramowitz, M. and Stegun, I. A.: Handbook of Mathematical Functions, Dover Publications, New-York, 1970.

Baines, P. G.: Topographic effects in Stratified flows, Cambridge Univ. Press, New York, 1995.

Davis, K. S.: Flow of Nonuniformly Stratified Fluid of Large Depth over Topography, M.Sc thesis in Mechanical Engineering, MIT, Cambridge, MA, 1999.

Dewan, E. M., Picard, R. H., O’Neil, R. R., Gardiner, H. A., Gibson, J., Mill, J. D., Richards, E., Kendra, M., and Gallery, W. O.: MSX satellite observations of thunderstorm-generated gravity waves in mid-wave infrared images of the upper stratosphere, Geophys. Res. Lett., 25, 939-942, 1998.

Drazin, P. G.: On the steady flow of a fluid of variable density past an obstacle, Tellus, 13, 239-251, 1961.

Drazin, P. G. and Moore, D. W.: Steady two dimensional flow of fluid of variable density over an obstacle, J. Fluid. Mech., 28, 353-370, 1967.

Durran, D. R.: Two-Layer solutions to Long's equation for vertically propagating mountain waves, Quart. J. Roy. Meteorol. Soc., 118, 415-433, 1992.

Dutton, J. A.: The Ceaseless Wind, Dover Publications, New York, 1986.

Eckermann, S. D. and Preusse, P.: Global measurements of stratospheric mountain waves from space, Science, 286, 1534-1537, 1999.

Haagenson, P. L., Dudhia, J., Grell, G. A., and Stauffer, D. R.: The Penn State/NCAR mesoscale model(MM5), 1994, Source code documentation, NCAR Technical Note, NCAR/TN-392+STR, 1994.

Humi, M.: On the Solution of Long's Equation Over Terrain, Il Nuovo Cimento C, 27, 219-229, 2004a.

Humi, M.: Estimation of Atmospheric Structure Constants from Airplane Data, J. Atmos. Oceanic Technol., 21, 495-500, 2004b.
Humi, M.: On the Solution of Long's Equation with Shear, Siam J. App. Math, 66(6), 1839-1852, 2006.

Jumper, G. Y., Murphy, E. A., Ratkowski, A. J., and Vernin, J.: Multisensor Campaign to correlate atmospheric optical turbulence to gravity waves, AIAA paper, AIAA-2004-1077, 2004.

Lily, D. K. and Klemp, J. B.: The effect of terrain shape on nonlinear hydrostatic mountain waves, J. Fluid Mech., 95, 241-261, 1979.

Long, R. R.: Some aspects of the flow of stratified fluids I. Theoretical investigation, Tellus, 5, 42-57, 1953.

Long, R. R.: Some aspects of the flow of stratified fluids II. Theoretical investigation, Tellus, 6, 97-115, 1954.

Long, R. R.: Some aspects of the flow of stratified fluids III. Continuous density gradients, Tellus, 7, 341-357, 1955.

Long, R. R.: The motion of fluids with density stratification, J. Geophys. Res., 64, 2151-2163, 1959.

Nappo, C. J.: Atmospheric Gravity Waves, Academic Press, Boston, 2002.

Peltier, W. R. and Clark, T. L.: Nonlinear mountain waves in two and three spatial dimensions, Quart. J. Roy. Meteorol. Soc., 109, 527-548, 1983.

Shutts, G. J., Kitchen, M., and Hoare, P. H.: A large amplitude gravity wave in the lower stratosphere detected by radiosonde, Quart. J. Roy. Meteorol. Soc., 114, 579-594, 1988.

Smith, R. B.: Linear theory of stratified hydrostatic flow past an isolated mountain, Tellus, 32, 348-364, 1980.

Smith, R. B.: Hydrostatic airflow over mountains, Adv. Geophys., 31, 1-41, 1989.

Yih, C.-S.: Equations governing steady two-dimensional large amplitude motion of a stratified fluid, J. Fluid Mech., 29, 539-544, 1967.

Yih, C.-S.: Stratified flows, Academic Press, New York, NY, 1980. 